\title{
Application of Proximity Operator in the MR Image Segmentation
}

\author{
Dongsheng $\mathrm{Ji}^{1,2}$, Yukai Yao ${ }^{1}$, Qingjun Yang ${ }^{1}$, Kaiming Liao ${ }^{2}$ and Xiaoyun Chen ${ }^{1, *}$ \\ ${ }^{1}$ School of Information Science\& Engineering, Lanzhou University, Lanzhou, 730000, China; ${ }^{2}$ Gansu Radio \& TV Uni- \\ versity, LanZhou, 730000, China
}

\begin{abstract}
GAC approach to MR image segmentation method. Our method is a combination of geodesic active contours and the optimization tool of proximity operator in that it uses proximity operator to iteratively deform the contour. Consequently, it has the following advantages. The operator has the ability to jump over local minima and provide a more global result. The proximity operator is used to solve GAC fast image segmentation model, gets an efficient iterative algorithm. Our approach easily extends to the segmentation of MRI objects and not sensitive to the noise. In addition, the algorithm is suitable for interactive correction and is shown to always converge. Experimental results and analyses are provided.
\end{abstract}

Keywords: Geodesic active contours, image segmentation, MRI, proximity operator.

\section{INTRODUCTION}

MRI is a kind of more unobtrusive way of imaging. And short axis image for detection has high value in global and regional cardiac function, in order to effectively analysis heart function and 3-D reconstruction to accurately segmentation, left ventricular and right ventricular is necessary.

At present, there are multiple segmentation method of left ventricle, mostly based on variation method, such as Paragios [1] using variation method to realize automatic segmentation of left ventricle, ChenQiang [2] improved the Cemers [3] model to partition left ventricle contour. Variation method is, however, by solving the energy functional minimization to partition image, only get local minimum value, so this method is more sensitive to initial contour, robustness and stability are poor; Based on ASM [4] and AAM [5] are all belong to statistical deformable model, its advantage is that it allows a degree of shape changes and can constraint on behalf of one kind of shape, but its target search is depending on the initial point set, also easily trapped in local extreme. So for cardiac MR images uneven gray, left and right ventricle are very close with other organizations around the grayscale, has weak edge, edge fracture and noise caused edge blur etc. Phenomenon, these methods all exist edge leakage phenomenon, segmentation accuracy are affected. Geometric active contour [6] based on graph theory methods to map image for weighted undirected graph, the pixel as a node, after the initial contour is determined, according to the initial contour strip, the target contour segmentation problem is converted to find the problem of minimum cut sets in the area of the strip. Image segmentation problem can be converted to the global optimization

*Address correspondence to this author at the School of Information Science\& Engineering, Lanzhou University, Lanzhou, 730000, China; E-mails: chenxy@1zu.edu.cn, and jids8012@163.com problem in the method, in order to get the global optimal solution, but because of the MR image gray unequal, weak edge, edge fracture and noise phenomenon etc, for improve the segmentation accuracy, we introduce the shape statistics method to govern the evolution of the edge curve.

Based on the detailed analysis of the left ventricle MR image segmentation problem, in this paper, we propose the method that is geometric active contour based on graph theory combine with shape statistical.

Geometric active contour model (GAC) [3] is an effective target automatic segmentation method. It through some constraint conditions in high dimensional space of the outline of the initial iterative evolution, to realize the image segmentation. But as a result of echocardiogram heart valves edge and texture feature is not very prominent, and it's complicated structure, even if the geometric active contour segmentation is also very difficult. A kind of effective solution is of according to the specific object segmentation, make full use of prior knowledge of the target, and under the guidance of prior knowledge to partition MR image. For the intervention of prior knowledge, make by fuzzy, shade and noise interference and is difficult to deal with the image goal, specific target segmentation also reached an unprecedented accurate and efficient $[7,8]$.

This method on the basis of point distribution model through training form registration and mode analysis was carried out on the, with shapes statistics to constraints, the active contour model based on graph theory left ventricle MR image segmentation, effectively deal with curve evolution when the edge of the leakage problems, improve the segmentation accuracy.

In addition, The method not only can choose a different target or different initial contour of the same target, but also provides interactive segmentation result changes. 


\section{GAC MODEL BASED ON PRIOR SHAPE OF THE SAMPLE REGISTRATION AND MODEL ANALYSIS}

Because there are gray uneven in the left ventricle MR images, weak edge, edge fracture and the phenomenon such as noise, when the GAC based on graph theory division the left ventricle MR image will occur edge of the leak. In order to prevent leakage of curve edge, in this section, introduced statistics constraint curve shape $[9,10]$.

In this paper, the shape of the target knowledge representation into velocity field, embedded into the level set iterative equation, drive the zero level set evolve to the ideal contour. In this paper, the main work is in $\mathrm{C}-\mathrm{V}$ geometric active contour model based on,

$$
\frac{\partial \varphi}{\partial t}=u(x)\left(\mathrm{k}+v_{0}\right)|\nabla \varphi|+\nabla u \cdot \nabla \varphi, u(x)=-\left|\nabla G_{\sigma} * I\right|
$$

$v_{0}$ does not depend on outward expansion force contour evolution of the image; $k$ contour curvature; $u$ image gradient. Under the level set, surface function $\varphi$ in the elastic force, expansion force and image gradient force, driving the zero level evolution to the target edges.

Controlled by a priori knowledge is to add a new item in the equation of the contour evolution. New item prior knowledge with the original form new force, internal force and the external force driving outline to the ideal goal evolution. Additional prior knowledge of the force can be divided into two levels, one is the low level of regional restrictions, the zero level evolution in specific areas; another is the shape of the high level constraints, make zero convergence in specific prior shape. Hence the new iteration equation is obtained [11]:

$$
\frac{\partial \varphi}{\partial t}=u(x)\left(\mathrm{k}+v_{0}\right)|\nabla \varphi|+\nabla u \cdot \nabla \varphi+\sum F_{i} \bullet \nabla \varphi
$$

$F_{i}$, the target of a priori knowledge. The kind of direct, efficient speed force, but how to prior knowledge representation into can directly drive the evolution of zero velocity field is the key to the problem. Due to noise, motion blur and object and the background gray similarity, makes the segmentation error is difficult to avoid. In order to improve the segmentation accuracy, sometimes need to use in the process of segmentation of left ventricle prior shape knowledge. The velocity field of force near the target point had a trend to move to the target. For prior shape outline, define the point (including contour inside and outside points) to the $C$ distance $\mathcal{E}$ :

$\varepsilon(\mathrm{X})=\min \left(\left|\mathrm{X}-\mathrm{X}_{I}\right|\right), \mathrm{X}_{I} \in C$

Velocity field $\delta$ :

$F_{\text {shape }}(\mathrm{X})=f_{s}(\varepsilon) \frac{\nabla \varepsilon}{|\nabla \varepsilon|}$

$F_{\text {shape }}$ Pointing to a priori shapes on the near point, $f_{s}(\varepsilon)$ control size.
Prior shape velocity field force of nature is very important. There are two kinds of the nature of the force, a kind of similar to the elastic force, more far away from the prior shape, the stronger the field force; another kind similar to the electric field force, the closer distance prior shape, the greater field force. Hope $F_{\text {shape }}$ only to the neighbor points to work, and the closer distance, the greater the field force. Set farthest effectively distance:

$$
f_{s}(\varepsilon)= \begin{cases}k(\delta-\varepsilon) & \varepsilon \leq \delta \\ 0 & \varepsilon>\delta\end{cases}
$$

In the end, based on the prior shape of geometric active contour model

$$
\frac{\partial \varphi}{\partial t}=u(x)\left(\mathrm{k}+v_{0}\right)|\nabla \varphi|+\nabla u \cdot \nabla \varphi+f_{s}(\mathrm{~d}) \frac{\nabla \varepsilon}{|\nabla \varepsilon|} \cdot \nabla \varphi
$$

Through the following a variegated images based on transcendental GAC partition in the shape of the effectiveness of specific objectives. $\delta=15$. Gray images of the brain and abdominal MR images segmented by shape-based GAC in Fig. (1).

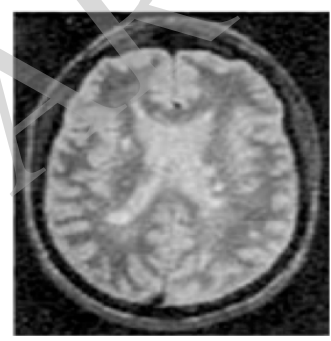

(a)

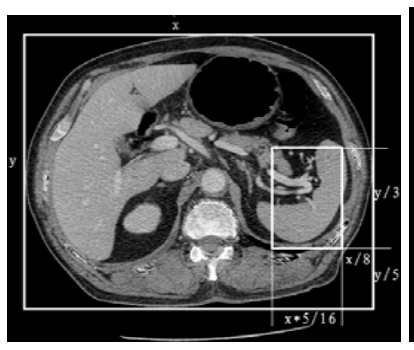

(c)

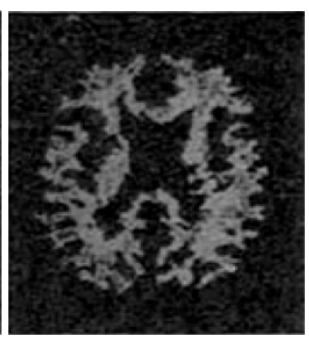

(b)

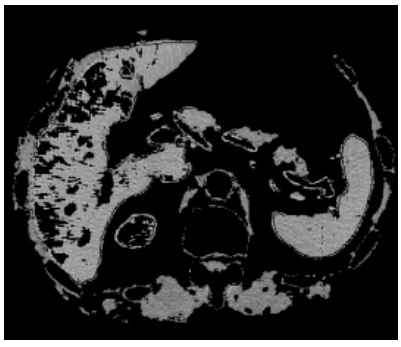

(d)
Fig. (1). Based on the transcendental GAC in the shape of the target segmentation. (a) Gray images of the brain by a bar and salt \& pepper noise; (b) Gray images of the brain segmented by GAC; (c) Abdominal MR images; (d) Abdominal MR images segmented by shape-prior-based GAC [12].

Active contours based on graph theory method to divide the entire black, this segmentation method is only interested in the elliptical target zone. Shape due to join the a priori constraints, makes the target by shade can also be a good segmentation, target shape of translation, rotation and scaling has no impact on the segmentation results, and the method also is not sensitive to noise.

Method of active contours based on graph theory and methods in this chapter in the same initial and outer contour segmentation of cardiac MR image segmentation, which reflects a priori shape plays a role in cardiac MR image segmentation in Fig. (2). 


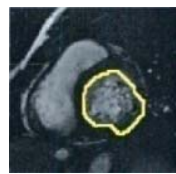

(a)

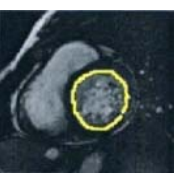

(b)

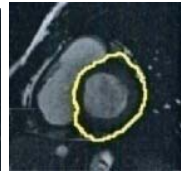

(c)

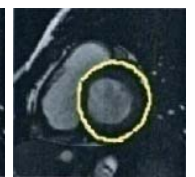

(d)
Fig. (2). Shape constraints on the result of segmentation.

Active contour segmentation method based on graph theory, edge leakage occurred when MR images of left ventricle (Fig. 2a-d), it is necessary to add shapes in the segmentation process statistical constraints [13, 14].

Based on the above training in the shape of a registration and change pattern analysis, this section proposes combining graph theory with the shape statistics of the left ventricle MR image segmentation algorithm is proposed. Namely in the process of evolution curve, must also will curve projection to allow space shape constraints imposed by the shape. Due to the boundary of the training sample is expressed with $\mathrm{N}$ points, and the curve of target segmentation active contour $\mathrm{C}$ is not necessarily the $\mathrm{N}$ points, So when calculating the shape change vector can't directly do bad. $d \widehat{x}_{i}=d \widehat{x}_{i} C, \mathrm{C}$, as the shape change of the point.

\section{PROXIMITY OPERATOR}

This paper based proximal operator [15] of forwardbackward splitting algorithm for solving the MR image segmentation model, given a new algorithm for constrained optimization problems.

We have described the signal recovery problem as that of inferring a signal $\bar{x}$ in a real Hilbert space $H$ from the observation of a signal $\mathrm{z}$ in a real Hilbert space $\mathrm{G}$. In this section, we consider the standard linear data formation model in which $\mathrm{z}$ is related to $\bar{x}$ via the model

$$
z=T \bar{x}+w
$$

where $\mathrm{T}: \mathrm{H} \rightarrow \mathrm{G}$ is a linear operator and where ${ }_{\mathrm{w}} \in \mathrm{G}$ stands for an additive noise perturbation.

Problem 1 Let H,G,K are all real Hilbert space.

(i) $\mathrm{k}$ be a real Hilbert space;

(ii) $\mathrm{T}: \mathrm{H} \rightarrow \mathrm{G}$ be a nonzero bounded linear operator;

(iii) $\mathrm{L}: \mathrm{H} \rightarrow \mathrm{K}$ be a objective bounded linear operator such that $L^{-1}=L^{*}$;

(iv) $\mathrm{f} \in \Gamma_{0}(K)$.

The objective is to

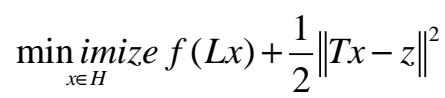

Let $\mathrm{g}$ be a real Hilbert space of lower semi-continuous convex function call operator

$$
\operatorname{prox}_{g}: H \rightarrow H: x \mid \rightarrow \underset{y \in H}{\arg \min } g(y)+\frac{1}{2}\|x-y\|_{2}^{2}
$$

Function approaching g operator (proximity operator). Algorithm based on the operator of an impending major result is given by the following theorem:

Theorem: Hypothetical question solution set of $\mathrm{G}$ is not empty, and $\left\{\gamma_{n}\right\}, n \in N$ is a sequence of positive real number and meet.

$0<\inf _{n \in N} \gamma_{n} \leq \sup _{n \in N} \gamma_{n}<\frac{2}{\|L\|^{2}}$

Define the following iterative,

$x_{n+1}=x_{n}+\lambda_{n}\left\{\left(T^{* \circ} \operatorname{prox\gamma }_{n} f^{\circ} T\right)\left[x_{n}-\gamma_{n}\left(L^{*}\left(L x_{n}-b\right)+c_{n}\right)\right]+a_{n}-x_{n}\right\}$

To conclude the establishment:

(1) $\left\{x_{n}\right\}_{n \in N}$ Weakly converges to the solution set $G$ of a point $x$;

(2) $\sum_{n \in N}\left\|L^{*} L\left(x_{n}-x\right)\right\|^{2}<+\infty$;

(3) $\sum_{n \in N}\left\|\left(T^{* \circ} \operatorname{prox}_{n} f^{\circ} T\right)\left[x_{n}-\gamma_{n}\left(L^{*}\left(L x_{n}-b\right)\right)\right]-x_{n}\right\|<+\infty$;

(4) $\left\{x_{n}\right\}_{n \in N}$ Strong converge $x$, and only if, $\lim d_{G}\left(x_{n}\right)=0$.

If, $\lambda_{n} \equiv 1, a_{n} \equiv 0, c_{n} \equiv 0$, Obviously these three series will meet the conditions of the theorem, The iteration formula

$x_{n+1}=\left(T^{* \circ} \operatorname{prox}_{n} f^{\circ} T\right)\left[x_{n}-\gamma_{n} L^{*}\left(L x_{n}-b\right)\right]$

is problem solution.

plify the question 1 , there are:

$\left(T^{* \circ} \operatorname{prox}_{n} f^{\circ} T\right)(x)=\operatorname{prox}_{\gamma_{n \mid \|_{1}}} x=\operatorname{sign}(x) \bullet \max \left\{|x|-\gamma_{n}, 0\right\}$

Known by its soft-threshold (soft--thresholding) or contraction (shrinkage) operator:

$$
\begin{aligned}
& \min _{x \in R^{N}} \gamma\|x\|_{1}+\frac{1}{2}\|L x-b\|_{2}^{2} \\
& x_{n+1}=\operatorname{prox}_{\gamma_{n \mid \|_{1}}}\left(x_{n}-\gamma_{n} L^{*}\left(L x_{n}-b\right)\right)
\end{aligned}
$$

Consider the constrained optimization problems 1, Lagrange form,

$$
\min _{x \in R^{N}} \gamma\|x\|_{1}+\frac{1}{2}\|A x-b\|_{2}^{2}
$$

Here, $A=U V^{-1}, x$ For image Wavelet coefficients, Optimization,

$$
x_{n+1}=\operatorname{prox}_{n\|\bullet\|_{1}}\left[x_{n}-\gamma_{n}\left(A^{*}\left(A x_{n}-b\right)\right)\right]
$$

Detailed algorithm pseudo code algorithm later, where $b$ is down-sampled at random $\mathrm{k}$-space data (can contain noise), 
$x_{0}$ is initial value, $x_{k+1}$ Segmentation of contours, immediately after the calculation of MR image segmentation.

With GAC model segmentation after left ventricular internal and external contours, approximation operator again for precise segmentation, to obtain the very good segmentation effect, especially on boundary integral effect is not obvious, as shown in Fig. (3).

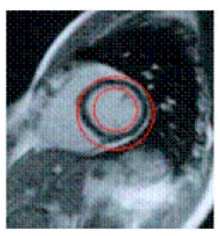

(a)

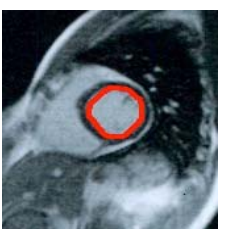

(b)

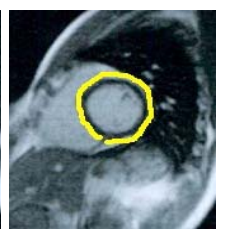

(c)
Fig. (3). Proximity Operator segmentation left ventricular external and internal profile.

\section{INTERACTIVE MODIFICATION OF SEGMENTA- TION RESULTS}

Image segmentation depends to a large extent on the subjective understanding of the image, in order to better address segmentation must also rely on other mechanisms, such as interacting with the user. Therefore, the results of interaction modification is necessary. Methods this chapter can provide interactive modification of the segmentation results. This interest in active contours based on graph theory methods have the flexibility to update the source point and the meeting point Fig. (4).

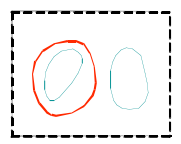

(a)

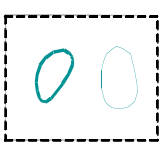

(b)

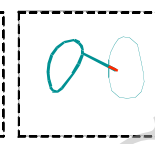

(c)

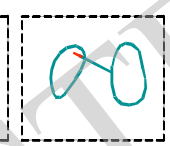

(d)
Fig. (4). Interactive modification of segmentation.

If the final result does not meet user needs, users can modify multiple times until it reaches the user satisfaction. This interactive modifications greatly facilitated humancomputer interaction, improve the segmentation accuracy.

\section{EXPERIMENTAL METHODS}

Fig. (5) for the method and document [12] methods of segmentation results. This method effective divided the left ventricle and capture larger area. Contour segmentation results can be seen from the left ventricle, the method with high robustness in this paper.

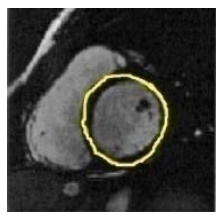

(a) Manual calibration

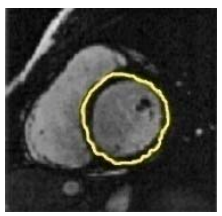

(b) Literature [14]

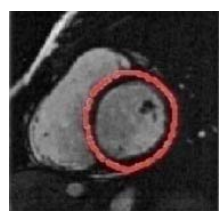

(c) Method in the paper
Fig. (5). comparison of the method and document [17].

We compare out results with those obtained using the traditional active contours (Fig. 6), specifically, the GVF snakes, which have a large capture range and can move into boundary concavities. We use the GVF implementation available at http://iacl.ece.jhu.edu/projects/gvf/. We select the parameters of the GVF snakes as follows: $\mu=0.1$, Iteration count $=200$ (for computing the gradient vector field), $\alpha=0.05, \beta=0, \gamma=1, \kappa=0.6, \mathrm{Dmin}=2$, and Dmax=4. shows the results of our approach and GVF Snakes approach for the same real images. Note that the initial contours provided for GVF Snakes are more accurate than these for improved GAC.

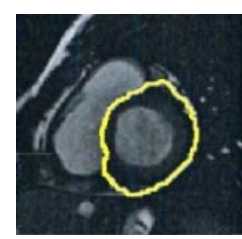

(a)

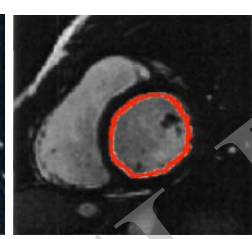

(b)
Fig. (6). The method comparison with GVF.

In this paper, some faults in a cardiac cycle of cardiac MR image segmentation. Fig. (7) for left ventricular internal profile contour segmentation results, Fig. (8) for left ventricular external profile contour segmentation results. Experimental show, the method in our paper has a better result for segmentation MR image.
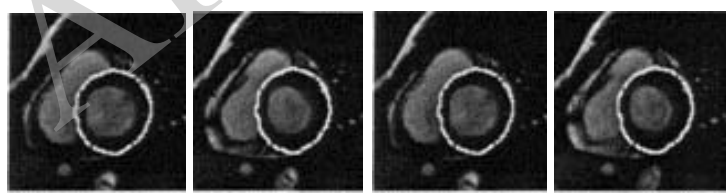

Fig. (7). Left ventricular internal profile segmentation results.
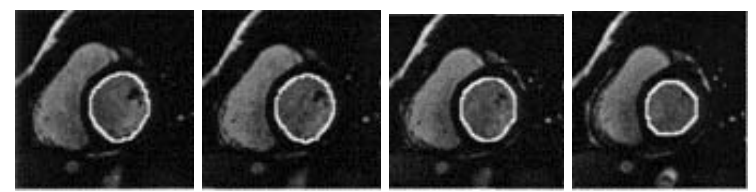

Fig. (8). Left ventricular external profile segmentation results.

\section{PERFORMANCE ANALYSIS}

In order to accurately evaluate the accuracy of this segmentation method, comparing the results with physician segmentation results by hand. MAD evaluation results with methods (pixels) shows in Table 1, MAD evaluation results with methods in the paper shows in Table 2.

Results from evaluation: method in the paper to achieve high levels of accuracy, the left ventricular internal and external contours with an average error of less than 1 pixel, The literature of [14-18] between the segmentation accuracy is 1-2 pixels and good stability than other methods in this chapter, segmentation error is the standard deviation of less than 0.2 .

\section{RESULTS AND DISCUSSION}

Active contours based on graph theory method is a new method of image segmentation, it maps the image for weighted undirected graph, as nodes pixels, converted in the vicinity of the initial contour contour segmentation problem solving min-cut problem within the band. This method con- 
Table 1. MAD evaluation results with methods (pixels).

\begin{tabular}{|c|c|c|c|}
\hline Method & Average & Variance & Maximum \\
\hline \hline Hybrid AAM [14] & 1.06 & 0.5 & 1.90 \\
\hline Shape particle filtering [15] & 1.10 & 0.3 & 0.7 \\
\hline Multi-view AAM [16] & 1.40 & 1.1 & 3.51 \\
\hline ASM [17] & 1.70 & & 1.34 \\
\hline Extended AAM [18] & 1.06 & & \\
\hline
\end{tabular}

Table 2. MAD evaluation results with methods in the paper (pixels).

\begin{tabular}{|c|c|c|c|}
\hline Method & Average & Variance & Maximum \\
\hline \hline Left ventricular external profile & 0.80 & 0.11 & 1.05 \\
\hline Left ventricular internal profile & 0.84 & 0.10 & 1.03 \\
\hline
\end{tabular}

verts the image segmentation problem for global optimization problems, so can get global optimal solution, but due to the presence in MR images of left ventricle papillary muscle disturbances, gray balance, weak, edge fracture and the noise fuzzy edges and other phenomena, still be marginal leakage phenomenon, in order to improve the segmentation accuracy statistics must also introduce shapes to constrain the evolution of curves.

For MR images of left ventricle segmentation problems, this chapter presents a combined active contour and shape of graph theory and statistics interactive segmentation of MR images of left ventricle. Taking into account active contour model based on graph theory methods split edges leak occurs when using statistics to constrain a shape based on graph theory, active contour segmentation of MR images of left ventricle. Point distribution model is used to describe the shape and training registration and change patterns of shapes. In the course of evolution curves, will Project to shape allows space to impose constraints. Prior shape constraints are due to join, making translation, rotation and scaling of the target shape has no effect on results, but not sensitive to noise. Experiments show that this method is valid for inner and outer contour segmentation of the left ventricle.

\section{CONFLICT OF INTEREST}

The authors confirm that this article content has no conflict of interest.

\section{ACKNOWLEDGEMENTS}

This paper is supported by Science and Technology Plan of GanSu Province (No.1308RJZA266). This support is gratefully acknowledged.

\section{REFERENCES}

[1] N. Paragios, "A varirtional approach for the segmentation of the left ventricle in MR cardiac image analysis," International Journal of Computer Vision, vol. 50, no. 3, pp. 345-362, 2002.
[2] Q. Chen, Z. Zhou, M. Tang, P.A. Heng, D.S. Xia, "Shape statistics variational approach for the outer contour segmentation of left ventricle MR images," IEEE Transactions on Information Technology in BioMedieine, vol. 10, no. 3, pp. 588-597, 2006.

[3] D. Cremers, F. Tischhauser, J. Weickert, C. Schnorr, "Diffusion snakes: introducing statistical shape knowledge into the MumfordShah functional," International Journal of Computer Vision, vol. 50, no. 3, pp. 295-313, 2002

[4] S.C. Mitchell, B.P.F. Lelieveldt, R.J. van der Geest, H.G Bosch, J.H.C. Reiver, M. Sonka, "Multistage hybrid active appearance model matching: segmentation of left and right ventricles in cardiac MR images," IEEE Transactions on Medical Imaging, vol. 20, no. 5, pp. 415-423, 2001.

[5] M. Kass, A. Witkin, D. T. Poulos, "Snake: active contour models," International Journal of Computer Vision, vol. 1, no. 4, pp. 321$331,1988$.

[6] N. Xu, R. Bansal, N. Ahuja, "Object segmentation using graph cuts based active contours," In: Proceedings of the IEEE Conference on Computer Vision and Pattern Recognition, Madison, Wl, 16-22 June 2003, vol. 2, pp. 46-53

[7] P.F. Felzenszwalb, D.P. Huttenlocher, "Efficient graph-based image segmentation," International Journal of Computer Vision, vol. 59, no. 2, pp. 167-181, 2004.

[8] S. Osher, J. Sethian, "Fronts propagating with curvature dependent speed: algorithms based on hamilton-jacobi formulations," Journal of Computational Physics, vol. 79, pp. 12-49, 1988.

[9] N. Paragios, O. Mellina-Gottardo, V. Ramesh, "Gradient vector flow fast geodesic active contours," In: The $8^{\text {th }}$ ICCV, vol. 1, pp. 67-73, 2001.

[10] J. Sethian, "Level set methods and fast marching methods," Cambridge University Press, $2^{\text {nd }}$ ed., 1999.

[11] D.D. Giusto, F. Massidda, C. Perra, "A fast algorithm for video segmentation and object tracking," In: International Conference on Digital Signal Processing (DSP 2002), Santorini, Greece, July 1-3, 2002.

[12] R. Goldenberg, R. Kimmel, E. Rivlin, M. Rudzsky, "Cortex segmentation-a fast variational geometric approach," In: IEEE Workshop on Variational and Level Set Methods (VLSM'01), July 13, Vancouver, Canada, 2001.

[13] P.L. Combettes, V.R. Wajs, "Signal recovery by proximal forwardbackward splitting," SLAM Journal on Multisc Modeling and Simulation, vol. 4, no. 4, pp. 1168-1200, 2005.

[14] B.P.F. Lelieveldt, R.J. van der Geest, J.H.C. Reiber, M. Sonka, "Multi-view active appearance models for consistent segmentation of multiple standard views: Application to long-and short-axis cardiac MR images," In: International Congress Series, vol. 1256, pp. 1141-1146, 2003. 
[15] Q. Chen, Z. Zhou, M. Tang, P.A. Heng, D.S. Xia, "Shape statistics variation approach for the outer contour segmentation of left ventricle MR images," IEEE Transactions on Information Technology in BioMedieine, vol. 10, no. 3, pp. 588-597, 2006.

[16] S.C. Mitchell, B.P.E. Lelieveldt, R.J. van der Geest, H,G. Bosch, J.H.C. Reiver, M. Sonka, "Multistage hybrid active appearance model matching: segmentation of left and right ventricles in cardiac MR images," IEEE Transactions on Medical Imaging, vol. 20, no. 5, pp. 415-423, 2001.
[17] M. de Bruijne, M. Nielsen, "Shape particle filtering for image segmentation," In: MICCAI2004, France, 26-30 Sept., 2004 , LNCS, vol. 3216, pp. 168-175.

[18] M.B. Stegmann, R. Fisker, B.K. Ersboll, "Extending and applying active appearance models for automated, high Precision segmentation in different image modalities," In: Proceedings of the $12^{\text {th }}$ Scandinavian Conference on Image Analysis, Bergen, Norway, June 2001, pp. 90-97.

Received: September 16, 2014

Revised: December 23, 2014

Accepted: December 31, 2014

() Ji et al.; Licensee Bentham Open.

This is an open access article licensed under the terms of the Creative Commons Attribution Non-Commercial License (http://creativecommons.org/licenses/by-nc/3.0/) which permits unrestricted, non-commercial use, distribution and reproduction in any medium, provided the work is properly cited. 